\title{
Two Bianchi Type VIII Spatially Homogeneous Cosmologies ${ }^{\star}$
}

\author{
J. G. Miller \\ Department of Physics, University of Utah, Salt Lake City, Utah 84112, USA
}

\begin{abstract}
We study two Bianchi type VIII analogues of Taub space and maximal analytic extensions of them. The first one has $\operatorname{SL}(2, R)$ as an isometry group, which acts transitively on spacelike hypersurfaces. The maximal extension has all of the pathological features of Taub-NUT space. The second one has the universal covering group of $\operatorname{SL}(2, R)$ as an isometry group. The maximal extension of the latter does not have these pathological properties and is geodesically complete.
\end{abstract}

\section{Local Solutions of the Einstein-Maxwell Equations}

Local solutions of the Einstein-Maxwell vacuum field equations have been derived by Carter [1] under the condition that the metrics admit a two-dimensional abelian isometry group and that the Hamilton-Jacobi equation for the geodesics and the Schrödinger equation separate in certain coordinate systems. The metrics contain several parameters and when some of the parameters are zero, the metrics admit a four-dimensional local isometry group. It is our aim to study two of these metrics that admit a four-dimensional local isometry group and show that they are special Bianchi type VIII spatially homogeneous cosmologies. Bianchi's classification of three-dimensional real Lie algebras into nine types is given by Taub [2]. Since the two metrics are Bianchi type VIII analogues of the Bianchi type IX metric discovered by Taub [2], we first review the known facts about the latter metric [3]. We will compare Taub space with the two Bianchi type VIII metrics throughout the paper.

The three metrics under consideration in this paper are all special cases of Carter's $[1]$ metrics listed in class $[\tilde{B}(+)]$. We first consider the "charged" TaubNut metric [4] given by

$$
\begin{aligned}
g= & \left(t^{2}+l^{2}\right)\left(d \theta^{2}+\sin ^{2} \theta d \phi^{2}\right)-4 l^{2} \Delta\left(t^{2}+l^{2}\right)^{-1}(d \psi+\cos \theta d \phi)^{2} \\
& +\left(t^{2}+l^{2}\right) \Delta^{-1} d t^{2},
\end{aligned}
$$

* Work supported in part by NSF Grant MPS 74-16311 AO1 
where

$$
\Delta \equiv t^{2}-2 m t-l^{2}+e^{2}
$$

and $m$ and $l$ are the Schwarzschild and NUT parameters, respectively, and $e$ is the electromagnetic parameter. The electromagnetic field tensor is obtained from the vector potential

$$
A=e\left(\sin \alpha+2 l t\left(t^{2}+l^{2}\right)^{-1} \cos \alpha\right)(d \psi+\cos \theta d \phi),
$$

where $\alpha$ is the complexion angle of the electromagnetic field. Linearly independent Killing vector fields are given by

$$
\begin{aligned}
Y_{1} & =\cos \phi \partial_{\theta}-\cot \theta \sin \phi \partial_{\phi}+\csc \theta \sin \phi \partial_{\psi}, \\
Y_{2} & =\sin \phi \partial_{\theta}+\cot \theta \cos \phi \partial_{\phi}-\csc \theta \cos \phi \partial_{\psi}, \\
Y_{3} & =\partial_{\phi}, \\
X_{3} & =\partial_{\psi}
\end{aligned}
$$

and satisfy commutation relations

$$
\left[Y_{i}, Y_{j}\right]=-C_{i j}^{k} Y_{k}, \quad\left[X_{3}, Y_{i}\right]=0,
$$

where

$$
C_{23}^{1}=-C_{32}^{1}=1, \quad C_{31}^{2}=-C_{13}^{2}=1, \quad C_{12}^{3}=-C_{21}^{3}=1
$$

are the structure constants of $\mathrm{SO}(3)$ or $\mathrm{SU}(2)$. The action of the local isometry group generated by $Y_{1}, Y_{2}$, and $Y_{3}$ is transitive on surfaces of constant $t$.

There are coordinate singularities at $\theta=0, \pi$, where the determinant of the metric components is zero, and at the zeros of $\Delta$ given by

$$
t_{ \pm} \equiv m \pm\left(m^{2}+l^{2}-e^{2}\right)^{1 / 2} \text {. }
$$

We assume that $e^{2}<m^{2}+l^{2}$ so that the roots are real and distinct.

The second metric is given by

$$
\begin{aligned}
g=\left(t^{2}\right. & \left.+l^{2}\right)\left(d \theta^{2}+\sinh ^{2} \theta d \phi^{2}\right)+4 l^{2} \Delta\left(t^{2}+l^{2}\right)^{-1}(d \psi+\cosh \theta d \phi)^{2} \\
& -\left(t^{2}+l^{2}\right) \Delta^{-1} d t^{2},
\end{aligned}
$$

where

$$
\Delta \equiv t^{2}-2 m t-l^{2}-e^{2}
$$

and the vector potential for the electromagnetic field is given by

$$
A=e\left(\sin \alpha+2 l t\left(t^{2}+l^{2}\right)^{-1} \cos \alpha\right)(d \psi+\cosh \theta d \phi) .
$$

Linearly independent Killing vector fields are given by

$$
\begin{aligned}
Y_{1} & =\cos \phi \partial_{\theta}-\operatorname{coth} \theta \sin \phi \partial_{\phi}+\operatorname{csch} \theta \sin \phi \partial_{\psi}, \\
Y_{2} & =\sin \phi \partial_{\theta}+\operatorname{coth} \theta \cos \phi \partial_{\phi}-\operatorname{csch} \theta \cos \phi \partial_{\psi}, \\
Y_{3} & =\partial_{\phi}, \\
X_{3} & =\partial_{\psi}
\end{aligned}
$$


and satisfy commutation relations

$$
\left[Y_{i}, Y_{j}\right]=-C_{i j}^{k} Y_{k}, \quad\left[X_{3}, Y_{i}\right]=0,
$$

where

$$
C_{23}^{1}=-C_{32}^{1}=1, \quad C_{31}^{2}=-C_{13}^{2}=1, \quad C_{12}^{3}=-C_{21}^{3}=-1
$$

are the structure constants of the Lorentz group in three dimensions. The local isometry group generated by $Y_{1}, Y_{2}$ and $Y_{3}$ is transitive on surfaces of constant $t$. There are coordinate singularities at $\theta=0, \pi$ and the zeros of $\Delta$ given by

$$
t_{ \pm} \equiv m \pm\left(m^{2}+l^{2}+e^{2}\right)^{1 / 2} .
$$

In this case, there is no restriction on $e$ and the roots are always real and distinct.

The third metric is given by

$$
\begin{aligned}
g= & \left(t^{2}+l^{2}\right)\left(d \theta^{2}+\cosh ^{2} \theta d \phi^{2}\right)+4 l^{2} \Delta\left(t^{2}+l^{2}\right)^{-1}(d \psi-\sinh \theta d \phi)^{2} \\
& -\left(t^{2}+l^{2}\right) \Delta^{-1} d t^{2},
\end{aligned}
$$

where $\Delta$ is given by (9) and

$$
A=e\left(\sin \alpha+2 l t\left(t^{2}+l^{2}\right)^{-1} \cos \alpha\right)(\mathrm{d} \psi-\sinh \theta d \phi) .
$$

Linearly independent Killing vector fields are given by

$$
\begin{aligned}
Y_{1} & =\partial_{\phi}, \\
Y_{2} & =\cosh \phi \partial_{\theta}-\tanh \theta \sinh \phi \partial_{\phi}+\operatorname{sech} \theta \sinh \phi \partial_{\psi}, \\
Y_{3} & =\sinh \phi \partial_{\theta}-\tanh \theta \cosh \phi \partial_{\phi}+\operatorname{sech} \theta \cosh \phi \partial_{\psi}, \\
X_{3} & =\partial_{\psi}
\end{aligned}
$$

and the satisfy the same commutation relations as (11). Likewise, the local isometry group generated by $Y_{1}, Y_{2}$, and $Y_{3}$ is transitive on surfaces of constant $t$. Metric (15) has coordinate singularities only at the zeros of $\Delta$ given by (14). All three metrics are of type D in the Petrov classification [1]. Having discussed the local properties of these metrics, we now turn to their global properties.

\section{Global Solutions}

We will consider the three metrics (1), (8), and (15) in turn. The Taub-NUT metric [Eq. (1)] has been discussed extensively in the literature [3, 5-7]. It is customary to take the surfaces of constant $t$ as three spheres, the topology of $\mathrm{SU}(2)$, and obtain all other possibilities by identification [5]. SU(2) is the two-fold universal covering group of $\mathrm{SO}(3)$. $\mathrm{SU}(2)$ is given by the set of matrices

$$
\left(\begin{array}{ll}
w+i x & i y-z \\
i y+z & w-i x
\end{array}\right)
$$


with $w^{2}+x^{2}+y^{2}+z^{2}=1$. Euler angle coordinates on $\mathrm{SU}(2)$ are defined by

$$
\begin{aligned}
& w=\cos \theta / 2 \cos (\phi=\psi) / 2, \\
& x=\sin \theta / 2 \cos (\phi-\psi) / 2, \\
& y=\sin \theta / 2 \sin (\phi-\psi) / 2, \\
& z=\cos \theta / 2 \sin (\phi=\psi) / 2
\end{aligned}
$$

and left-invariant 1 -forms on SU(2) in Euler angle coordinates are given by

$$
\begin{aligned}
& \omega^{1}=\cos \psi d \theta+\sin \theta \sin \psi d \phi, \\
& \omega^{2}=-\sin \psi d \theta+\sin \theta \cos \psi d \phi, \\
& \omega^{3}=d \psi+\cos \theta d \phi .
\end{aligned}
$$

These 1-forms satisfy $d \omega^{k}=-\frac{1}{2} C_{i j}^{k} \omega^{i} \wedge \omega^{j}$, where the $C_{i j}^{k}$ are the same as in (6). We can now express metric (1) and vector potential (3) in the global basis $\omega^{i}$ and obtain

$$
g=\left(t^{2}+l^{2}\right)\left(\left(\omega^{1}\right)^{2}+\left(\omega^{2}\right)^{2}\right)-4 l^{2} \Delta\left(t^{2}+l^{2}\right)^{-1}\left(\omega^{3}\right)^{2}+\left(t^{2}+l^{2}\right) \Delta^{-1} d t^{2}
$$

and

$$
A=e\left(\sin \alpha+2 l t\left(t^{2}+l^{2}\right)^{-1} \cos \alpha\right) \omega^{3} .
$$

When $\Delta<0$, the homogeneous surfaces of constant $t$ are spacelike and when $\Delta>0$, they are timelike. The $Y_{i}$ in (4) are right-invariant vector fields on $\mathrm{SU}(2)$, so $L_{Y_{i}} \omega^{j}=0$, and $X_{3}$ is a Killing vector field because the functions of $t$ before $\left(\omega^{1}\right)^{2}$ and $\left(\omega^{2}\right)^{2}$ are equal. If we let $X_{i}$ be the left-invariant vector fields on $\operatorname{SU}(2)$ that are dual to the $\omega^{i}$, then in Euler angle coordinates

$$
\begin{aligned}
& X_{1}=\cos \psi \partial_{\theta}+\csc \theta \sin \psi \partial_{\phi}-\cot \theta \sin \psi \partial_{\psi}, \\
& X_{2}=-\sin \psi \partial_{\theta}+\csc \theta \cos \psi \partial_{\phi}-\cot \theta \cos \psi \partial_{\psi}, \\
& X_{3}=\partial_{\psi} .
\end{aligned}
$$

The singularities in metric (1) at $\theta=0, \pi$ are just the degeneracies of Euler angle coordinates on $S^{3}$. We will denote the three disjoint regions of metric (20) by

$$
\begin{gathered}
\text { I, when } t>t_{+}, \\
\text {II, when } t_{-}<t<t_{+}, \\
\text {III, when } t<t_{-} .
\end{gathered}
$$

We are now left with the coordinate singularities at the zeros of $\Delta$. These singularities have been dealt with by Hawking and Ellis [3] by means of a principal fibre bundle closely related to the Hopf fibering of $S^{3}$ over $S^{2}$. Since $\operatorname{SO}(2)$ is a closed subgroup of $\mathrm{SU}(2), \mathrm{SU}(2) \approx S^{3}$ is a principal fibre bundle over $\mathrm{SU}(2)$ / $\mathrm{SO}(2) \approx S^{2}$ with fibre $\mathrm{SO}(2) \approx S^{1}$. The canonical projection map $\pi: S^{3} \rightarrow S^{2}$ is given by $(w, x, y, z) \leadsto\left(2(w x-y z), 2(w y+x z), w^{2}-x^{2}-y^{2}+z^{2}\right)=(\sin \theta \cos \phi, \sin \theta \sin \phi$, $\cos \theta$ ) and the vertical vector field $X_{3}$ is a fundamental vector field [8].

Hawking and Ellis considered $S^{3} \times R$ as a principal fibre bundle over $S^{2}$ with fibre $S^{1} \times R$ and we denote the canonical projection map again by $\pi: S^{3} \times R \rightarrow S^{2}$. 


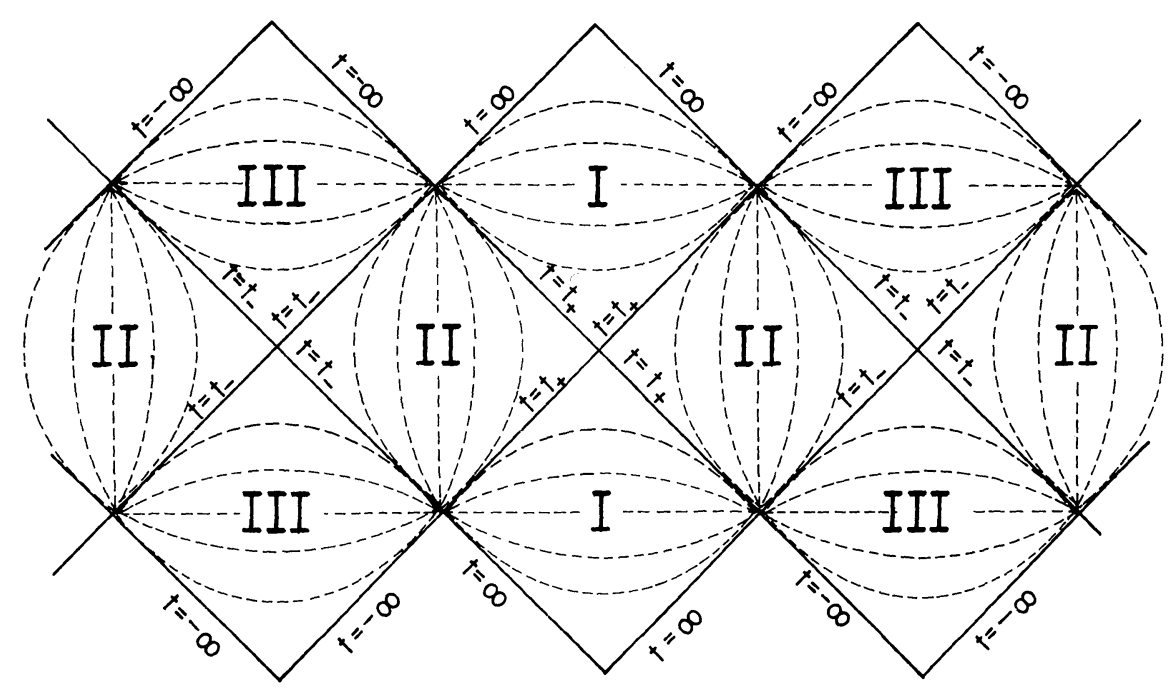

Fig. 1. The Penrose diagram for the maximal extension of metric (1), (8), and (15). The $\left(u_{+}, v_{+}\right)$coordinate system covers the squares consisting of two blocks labeled I and two blocks labeled II and the $\left(u_{-}, v_{-}\right)$coordinate system covers the squares consisting of two blocks labeled II and two blocks labeled III. Every null cone in the two-dimensional surface represented by the Penrose diagram is $\pm 45^{\circ}$ with the horizontal direction. The dashed lines are timelike and spacelike orbits of $X_{3}$ and the solid lines are bifurcate Killing horizons (null orbits of $X_{3}$ ). For metric (1), time flows from left to right and region II is a Bianchi type IX spatially homogeneous cosmology (Taub space). For metric (8) and (15), time flows from bottom to top and regions I and III are Bianchi type VIII spatially homogeneous cosmologies. For metrics (1) and (8), the orbits of $X_{3}$ are closed because of the identification $U_{\lambda}(p)=U_{\lambda+4 \pi}(p)$, where $U_{\lambda}$ is the flow of $X_{3}$. For metric (15), the orbits of $X_{3}$ are open and the extended manifold is geodesically complete

Then $X_{3}$ and $X_{4}=\partial_{t}$ are fundamental vector fields on $S^{3} \times R$, where $t$ is the coordinate on $R$. A connection in $S^{3} \times R$ was defined by taking $X_{1}$ and $X_{2}$ as horizontal vector fields. If we also let $\psi$ be the coordinate on $S^{1}$ with period $4 \pi$, then the connection form $\omega$ is given by

$$
\omega\left(X_{1}\right)=0, \quad \omega\left(X_{2}\right)=0, \quad \omega\left(X_{3}\right)=\partial_{\psi}, \quad \omega\left(X_{4}\right)=\partial_{t}
$$

where $\partial_{\psi}$ and $\partial_{t}$ are left-invariant vector fields on $S^{1} \times R$. The connection form is an equivariant, Lie algebra valued 1-form that annihilates the horizontal subspaces. Hawking and Ellis decomposed the Taub-NUT metric (20) as follows:

$$
g(X, Y)=g_{H}\left(\pi_{*} X, \pi_{*} Y\right)+g_{V}(\omega(X), \omega(Y)),
$$

where $X$ and $Y$ are tangent vectors to $S^{3} \times R, g_{H}$ is the metric of constant positive curvature on $S^{2}$ which in spherical coordinates is given by

$$
g_{H}=\left(t^{2}+l^{2}\right)\left(d \theta^{2}+\sin ^{2} \theta d \phi^{2}\right)
$$

and $g_{V}$ is the metric on $S^{1} \times R$ given by

$$
g_{V}=-4 l^{2} \Delta\left(t^{2}+l^{2}\right)^{-1} d \psi^{2}+\left(t^{2}+l^{2}\right) \Delta^{-1} d t^{2} .
$$


The type of singularity at the zeros of $\Delta$ in metric (27) is well known and corresponds to bifurcate Killing horizons. We consider Kruskal-like transformations [9]

$$
\begin{aligned}
& u_{ \pm}=\left|t-t_{ \pm}\right|^{1 / 2}\left|t-t_{\mp}\right|^{\kappa_{ \pm} / 2 \kappa_{\mp}} e^{\kappa_{ \pm} t} \cosh 2 l \kappa_{ \pm} \psi, \\
& v_{ \pm}=\left|t-t_{ \pm}\right|^{1 / 2}\left(t-\left.t_{\mp}\right|^{\kappa_{ \pm} / 2 \kappa_{\mp}} e^{\kappa_{ \pm} t} \sinh 2 l \kappa_{ \pm} \psi,\right.
\end{aligned}
$$

where

$$
2 \kappa_{ \pm} \equiv\left(t_{ \pm}-t_{\mp}\right) /\left(t_{ \pm}\left(t_{ \pm}-t_{\mp}\right)+e^{2}\right) \text {. }
$$

Each transformation maps region II $\left(t_{-}<t<t_{+}\right)$onto the quadrant $\left|v_{ \pm}\right|<u_{ \pm}$. In Kruskal-like coordinates, metric (27) becomes

$$
g_{V}=f_{ \pm}^{2}\left(d v_{ \pm}^{2}-d u_{ \pm}^{2}\right),
$$

where

$$
f_{ \pm}^{2} \equiv \kappa_{ \pm}^{-2}\left(t^{2}+l^{2}\right)^{-1}\left|t-t_{\mp}\right|^{1-\kappa_{ \pm} / \kappa_{\mp}} e^{-2 \kappa_{ \pm} t}
$$

is an analytic function of $u_{ \pm}^{2}-v_{ \pm}^{2}$. Both metrics (30) immediately extend analytically to the entire $\left(u_{ \pm}, v_{ \pm}\right)$plane, where $t_{-}<t<\infty$ for the upper sign and $-\infty<t<t_{+}$for the lower sign. The bifurcate Killing horizon corresponding to the $t_{+}$zero of $\Delta$ is given by $v_{+}= \pm u_{+}$and the bifurcate horizon corresponding to $t_{-}$ is given by $v_{-}= \pm u_{-}$. These metrics patch together as indicated in the Penrose diagram (Fig. 1) to give a maximal analytic extension of metric (27) and (25). The orbits of $\partial_{\psi}=2 l \kappa_{ \pm}\left(v_{ \pm} \partial_{u_{ \pm}}+u_{ \pm} \partial_{v_{ \pm}}\right)$are hyperbolas in the $\left(u_{ \pm}, v_{ \pm}\right)$plane. Since $\psi$ is periodic with period $4 \pi$, the points in the $\left(u_{ \pm}, v_{ \pm}\right)$plane are identified according to $U_{\lambda}(p)=U_{\lambda+4 \pi}(p)$, where $U_{\lambda}$ is the flow of $2 \kappa_{ \pm}\left(v_{ \pm} \partial_{u_{ \pm}}+u_{ \pm} \partial_{v_{ \pm}}\right)$. This identification means that the extended manifold is non-Hausdorff and that the points $\left(u_{ \pm}, v_{ \pm}\right)=(0,0)$ are not regular points of the manifold $[3,7]$.

We now discuss the second metric (8). The Lie group $\operatorname{SL}(2, R)$ is the Bianchi type VIII analogue of the Bianchi type IX group SU(2). SL(2, $R$ ) is the two-fold covering group of the identity component of the Lorentz group in three dimensions. $\operatorname{SL}(2, R)$ is given by the set of matrices

$$
\left(\begin{array}{cc}
w+x & y-z \\
y+z & w-x
\end{array}\right)
$$

with $w^{2}-x^{2}-y^{2}+z^{2}=1$ and coordinates similar to Euler angle coordinates are given by

$$
\begin{aligned}
& w=\cosh \theta / 2 \cos (\phi+\psi) / 2, \\
& x=\sinh \theta / 2 \cos (\phi-\psi) / 2, \\
& y=\sinh \theta / 2 \sin (\phi-\psi) / 2, \\
& z=\cosh \theta / 2 \sin (\varphi+\psi) / 2 .
\end{aligned}
$$

Left-invariant 1 -forms on $\operatorname{SL}(2, R)$ in these coordinates are given by

$$
\begin{aligned}
& \omega^{1}=\cos \psi d \theta+\sinh \theta \sin \psi d \phi, \\
& \omega^{2}=-\sin \psi d \theta+\sinh \theta \cos \psi d \phi, \\
& \omega^{3}=d \psi+\cosh \theta d \phi .
\end{aligned}
$$


These 1-forms satisfy $d \omega^{k}=-\frac{1}{2} C_{i j}^{k} \omega^{i} \wedge \omega^{j}$, where the $C_{i j}^{k}$ are the structure constants of SL $(2, R)$ and are given by (13). We now express metric (8) and vector potential (10) in the global basis $\omega^{i}$ and find that

$$
g=\left(t^{2}+l^{2}\right)\left(\left(\omega^{1}\right)^{2}+\left(\omega^{2}\right)^{2}\right)+4 l^{2} \Delta\left(t^{2}+l^{2}\right)^{-1}\left(\omega^{3}\right)^{2}-\left(t^{2}+l^{2}\right) \Delta^{-1} d t^{2}
$$

and

$$
A=e\left(\sin \alpha+2 l t\left({ }^{2}+l^{2}\right)^{-1} \cos \alpha\right) \omega^{3} .
$$

When $\Delta>0$, the homogeneous hypersurfaces of constant $t$ are spacelike and when $\Delta<0$, they are timelike. The $Y_{i}$ in (11) are right-invariant vector fields on $\operatorname{SL}(2, R)$. If we let $X_{i}$ be the left-invariant vector fields on $\operatorname{SL}(2, R)$ dual to the $\omega^{i}$, then

$$
\begin{aligned}
& X_{1}=\cos \psi \partial_{\theta}+\operatorname{csch} \theta \sin \psi \partial_{\phi}-\operatorname{coth} \theta \sin \psi \partial_{\psi}, \\
& X_{2}=-\sin \psi \partial_{\theta}+\operatorname{csch} \theta \cos \psi \partial_{\phi}-\operatorname{coth} \theta \cos \psi \partial_{\psi}, \\
& X_{3}=\partial_{\psi} .
\end{aligned}
$$

The coordinate singularities at $\theta=0, \pi$ are due to degeneracies in the coordinate system. We will denote the three disjoint regions of metric (34) by (23), where $t_{ \pm}$ is now given by (14) instead of (7).

We are now left with coordinate singularities at the zeros of $\Delta$. Since $\mathrm{SO}(2)$ is a closed subgroup of $\operatorname{SL}(2, R), \operatorname{SL}(2, R) \approx L^{3} \equiv\left\{(w, x, y, z) \in R^{4} \mid w^{2}-x^{2}-y^{2}+z^{2}=1\right\}$ is a principal fibre bundle over $\operatorname{SL}(2, R) / \mathrm{SO}(2) \approx H^{2} \equiv\left\{\left(x_{1}, x_{2}, x_{3}\right) \in R^{3} \mid x_{1}^{2}+x_{2}^{2}-\right.$ $\left.x_{3}^{2}=-1\right\}$ with fibre $\mathrm{SO}(2) \approx S^{1}$. The canonical projection map $\pi: L^{3} \rightarrow H^{2}$ is given by $(w, x, y, z) m\left(2(w x-y z), 2(w y+x z), w^{2}+x^{2}+y^{2}+z^{2}\right)=(\sinh \theta \cos \phi, \sin \theta \sin \phi$, $\cosh \theta$ ) and the vertical field $X_{3}$ is a fundamental vector field.

We consider $L^{3} \times R$ as a principal fibre bundle over $H^{2}$ with fibre $S^{1} \times R$ and we denote the canonical projection map again by $\pi: L^{3} \times R \rightarrow H^{2}$. Now $X_{3}$ and $X_{4}=\partial_{t}$ are fundamental vector fields on $L^{3} \times R$, where $t$ is the coordinate on $R$, and a connection in $L^{3} \times R$ is defined by taking $X_{1}$ and $X_{2}$ as horizontal vector fields. We decompose metric (34) as follows:

$$
g(X, Y)=g_{H}\left(\pi_{*} X, \pi_{*} Y\right)=g_{V}(\omega(X), \omega(Y)),
$$

where $X$ and $Y$ are tangent vectors to $L^{3} \times R, \omega$ is the connection 1-form, $g_{H}$ is the metric of constant negative curvature on $H^{2}$ which in pseudo-spherical coordinates is given by

$$
g_{H}=\left(t^{2}+l^{2}\right)\left(d \theta^{2}+\sinh ^{2} \theta d \phi^{2}\right)
$$

and $g_{V}$ is the metric on $S^{1} \times R$ given by

$$
g_{V}=4 l^{2} \Delta\left(t^{2}+l^{2}\right)^{-1} d \psi^{2}-\left(t^{2}+l^{2}\right) \Delta^{-1} d t^{2} .
$$

We again consider transformation (28) with $t_{ \pm}$given by (14) and $\kappa_{ \pm}$given by

$$
2 \kappa_{ \pm} \equiv\left(t_{ \pm}-t_{\mp}\right) /\left(t_{ \pm}\left(t_{ \pm}-t_{\mp}\right)-e^{2}\right) \text {. }
$$

Each transformation (28) maps region II $\left(t_{-}<t<t_{+}\right)$onto the quadrant $\left|v_{ \pm}\right|<u_{ \pm}$. In Kruskal-like coordinates, metric (39) becomes

$$
g_{V}=f_{ \pm}^{2}\left(d u_{ \pm}^{2}-d v_{ \pm}^{2}\right),
$$


where $f_{ \pm}^{2}$ is given by (31) with the appropriate $t_{ \pm}$and $\kappa_{ \pm}$. Both metrics (41) extend analytically to the entire $\left(u_{ \pm}, v_{ \pm}\right)$plane and patch together as indicated in the Penrose diagram (Fig. 1) to give a maximal extension of metric (39) and (37). The orbits of $X_{3}$ are periodic with period $4 \pi$ and the extension is nonHausdorff.

We now discuss the third metric (15). It turns out that this metric is already expressed in what is called canonical coordinates of the second kind [10] on $\operatorname{SL}(2, R)$, which are given by

$$
\begin{aligned}
& w=\cosh \theta / 2 \cosh \phi / 2 \cos \psi / 2+\sinh \theta / 2 \sinh \phi / 2 \sin \psi / 2, \\
& x=\cosh \theta / 2 \sinh \phi / 2 \cos \psi / 2+\sinh \theta / 2 \cosh \phi / 2 \sin \psi / 2, \\
& y=\sinh \theta / 2 \cosh \phi / 2 \cos \psi / 2-\cosh \theta / 2 \sinh \phi / 2 \sin \psi / 2, \\
& z=\cosh \theta / 2 \cosh \phi / 2 \sin \psi / 2-\sinh \theta / 2 \sinh \phi / 2 \cos \psi / 2
\end{aligned}
$$

The left-invariant 1 -forms (33) in these coordinates are given by

$$
\begin{aligned}
& \omega^{1}=\sin \psi d \theta+\cosh \theta \cos \psi d \phi, \\
& \omega^{2}=\cos \psi d \theta-\cosh \theta \sin \psi d \phi, \\
& \omega^{3}=d \psi-\sinh \theta d \phi
\end{aligned}
$$

and dual left-invariant vector fields (36) by

$$
\begin{aligned}
& X_{1}=\sin \psi \partial_{\theta}+\operatorname{sech} \theta \cos \psi \partial_{\phi}+\tanh \theta \cos \psi \partial_{\psi}, \\
& X_{2}=\cos \psi \partial_{\theta}-\operatorname{sech} \theta \sin \psi \partial_{\phi}-\tanh \theta \sin \psi \partial_{\psi}, \\
& X_{3}=\partial_{\psi} .
\end{aligned}
$$

The right-invariant vector fields (11) in these coordinates are given by (17). The range of the coordinates in (42) is $-\infty<\theta<\infty,-\infty<\phi<\infty, 0<\psi<4 \pi$ and $\psi$ is periodic with period $4 \pi$. The fibre bundle $L^{3} \times R \approx H^{2} \times S^{1} \times R \rightarrow H^{2}$ is trivial and the Lie group $\operatorname{SL}(2, R) \approx L^{3}$ is not simply connected. Instead of coordinates on $\operatorname{SL}(2, R)$, we take $(\theta, \phi, \psi)$ as a global coordinate system on $\operatorname{SL}(2, R)^{\sim} \approx R^{3}$, the universal covering group of $\operatorname{SL}(2, R)$. The range of $\psi$ is then from minus infinity to plus infinity. Metric (15) is defined on the Cartesian product of $\operatorname{SL}(2, R)^{\sim} \approx R^{3}$ with the disjoint intervals of the real line, $-\infty<t<t_{-}, t_{-}<t<t_{+}$ and $t_{+}<t<\infty$. Metric (15) and vector potential (16) can be expressed in terms of the global basis $\omega^{i}$ on $\operatorname{SL}(2, R)^{\sim}$ given by (43).

We consider again transformation (28) with $t_{ \pm}$given by (14) and $\kappa_{ \pm}$by (40). In Kurskal-like coordinates metric (15) becomes

$$
\begin{aligned}
g= & f_{ \pm}^{2}\left(d u_{ \pm}^{2}-d v_{ \pm}^{2}+4 l \kappa_{ \pm} \sinh \theta\left(u_{ \pm} d v_{ \pm}-v_{ \pm} d u_{ \pm}\right) d \phi\right. \\
& \left.-4 l^{2} \kappa_{ \pm}^{2} \sinh ^{2} \theta\left(u_{ \pm}^{2}-v_{ \pm}^{2}\right) d \phi^{2}\right)+\left(t^{2}+l^{2}\right)\left(d \theta^{2}+\cosh ^{2} \theta d \phi^{2}\right),
\end{aligned}
$$

where $f_{ \pm}^{2}$ is given by (31). Both metrics extend analytically to the entire $\left(u_{ \pm}, v_{ \pm}\right)$ plane and patch together as indicated in the Penrose diagram (Fig. 1) to give a geodesically complete Hausdorff extension of metric (15). The orbits of $X_{3}$ are open in this extension. 


\section{Geodesic Completeness}

Having found appropriate maximal extensions of the three local metrics, we now turn to the question of geodesic completeness. The extensions of metric (1) and (8) are incomplete with geodesics "approaching" $\left(u_{ \pm}, v_{ \pm}\right)=(0,0)$ with finite affine length. Thus we consider only the geodesics of metric (15) and prove that the extension is geodesically complete as claimed in Section 2. A Hamiltonian for the geodesic equations of metric (15) is given by

$$
H=\frac{1}{2}\left(\frac{p_{\theta}^{2}}{t^{2}+l^{2}}+\frac{\left(p_{\phi}+\sinh \theta p_{\psi}\right)^{2}}{\left(t^{2}+l^{2}\right) \cosh ^{2} \theta}+\frac{t^{2}+l^{2}}{4 l^{2} \Delta} p_{\psi}^{2}-\frac{\Delta}{t^{2}+l^{2}} p_{t}^{2}\right) .
$$

The Hamilton-Jacobi equation $\frac{\partial S}{\partial \lambda}+H=0$ separates in this coordinate system and we find that the action $S$ is given by

$$
S=-\varepsilon \lambda / 2+\alpha \phi+\beta \psi+\int^{\theta} \Theta^{1 / 2} d \theta+\int^{t} T^{1 / 2} \Delta^{-1} d t,
$$

where

$$
\begin{aligned}
& \Theta \equiv \kappa-(\alpha+\beta \sin \theta)^{2} / \cosh ^{2} \theta, \\
& T \equiv \beta^{2}\left(t^{2}+l^{2}\right)^{2} / 4 l^{2}+\Delta\left(\kappa-\varepsilon\left(t^{2}+l^{2}\right)\right)
\end{aligned}
$$

and $\varepsilon, \alpha, \beta$ and $\kappa$ are constants. We obtain the geodesics by quadratures:

$$
\begin{aligned}
& \lambda=-\int^{t} T^{-1 / 2}\left(t^{2}+l^{2}\right) d t, \\
& \phi=\int^{\theta} \frac{\alpha+\beta \sinh \theta}{\Theta^{1 / 2} \cosh ^{2} \theta} d \theta, \\
& \psi=\int^{\theta} \frac{\sinh \theta(\alpha+\beta \sinh \theta)}{\Theta^{1 / 2} \cosh ^{2} \theta} d \theta, \\
& \int^{\theta} \Theta^{-1 / 2} d \theta=-\int^{t} T^{-1 / 2} d t .
\end{aligned}
$$

The boundary at $t= \pm \infty$ in the Penrose diagram is at infinity in the sense that every geodesic that approaches this boundary has infinite affine length. This follows directly from (49) and the fact that $\lambda$ is an affine parameter. The incomplete geodesics in the $(\theta, \phi, \psi, t)$ coordinate system are approaching a zero of $\Delta$ and can be continued across a horizon. Hence the extension of metric (15) is geodesically complete.

\section{Conclusion}

We have studied two Bianchi type VIII analogues of Taub space. The one in which $\operatorname{SL}(2, R)$ is an isometry group is very similar to Taub space and the maximal analytic extension has the same pathological properties as Taub-NUT space $[3,11]$. The maximal analytic extension of the other Bianchi type VIII cosmology with $\operatorname{SL}(2, R)^{\sim}$ as an isometry group does not exhibit any of these pathologies and is geodesically complete. Of course, $\operatorname{SL}(2, R)=\operatorname{SL}(2, R)^{\sim} / N$, where $N$ is a discrete normal subgroup of $\operatorname{SL}(2, R)^{\sim}$ and there are other discrete normal sub- 
groups of $\operatorname{SL}(2, R)^{\sim}$ which we could have considered. What we have established is that metric (15) with $\psi$ periodic with period $4 \pi$ is globally isometric to metric (8) with $(\theta, \phi, \psi)$ as coordinates on $\operatorname{SL}(2, R)$ given by (32). An identification imposed on metric $(15)$ by considering $\operatorname{SL}(2, R)^{\sim} / N$ for some normal subgroup $N$ of $\operatorname{SL}(2, R)^{\sim}$ will lead to the same pathological properties of metric (8) if the orbits of $X_{3}$ become closed.

Very few geodesically complete solutions of the Einstein-Maxwell vacuum field equations are known. Many of the plane wave solutions are geodesically complete, but Penrose [12] has shown that they do not admit a global Cauchy hypersurface. The Killing horizons in the Bianchi type VIII cosmologies are also Cauchy horizons for the homogeneous spacelike hypersurfaces. The existence of a global Cauchy hypersurface has not been investigated for the geodesically complete extension.

The metrics (1), (8), and (15) have generalizations to include a Kerr parameter [1] and the Kerr-Taub-NUT metric has already been studied [13]. We expect similar results for the generalizations of metric (8) and (15).

\section{References}

1. Carter, B.: Commun. math. Phys. 10, 280 (1968)

2. Taub, A.: Ann. Math. 53, 472 (1951)

3. Hawking, S.W., Ellis, G.F.R.: The large scale structure of space-time. Cambridge: Cambridge University Press 1973

4. Brill, D.: Phys. Rev. B 133, 845 (1964)

5. Misner, C.: J. math. Phys. 4, 924 (1963)

6. Misner, C., Taub, A.: Zh. Eksp. Teor. Fiz. 55, 233 (1968) [Sov. Phys.-JETP 28, 122 (1969)]

7. Miller, J. G., Kruskal, M.D., Godfrey, B.: Phys. Rev. D 4, 2945 (1971)

8. Kobayashi, S., Nomizu, K.: Foundations of differential geometry, Vol. 1. New York-London: Interscience 1963

9. Walker, M.: J. math. Phys. 11, 2280 (1970)

10. Cohn,P.M.: Lie groups, pp. 110. Cambridge: Cambridge University Press 1957

11. Misner, C.: Taub-NUT space as a counterexample to almost anything. In: Lectures in Applied Mathematics, Vol. 8, pp. 160. Providence: American Mathematical Society 1967

12. Penrose, R.: Rev. Mod. Phys. 37, 215 (1965)

13. Miller, J. G.: J. math. Phys. 14, 486 (1973)

Communicated by R. Geroch

Received May 12, 1976

\section{Note Added in Proof}

S. T. C. Siklos has also considered metric (15) in a paper submitted to Physics Letters and has obtained the same maximal extension. 